\title{
Mise en évidence et dénombrement des Listeria à partir de produits laitiers
}

\author{
JL Bind \\ Laboratoire vétérinaire départemental, 46 avenue Gustave Eiffel, 37023 Tours Cedex, France
}

(Reçu le 19 mars 1990; accepté le 11 septembre 1990)

\begin{abstract}
Résumé - La systématisation des recherches de Listeria dans les produits pathologiques, alimentaires et notamment laitiers, amène à optimiser les techniques de mise en évidence et de dénombrement de ces germes.

Nous avons donc évalué parallèlement les performances respectives de la méthode officielle française "FDA modifiée", à partir des travaux de Lovett publiés en 1988 et celles de la méthode décrite par Van Netten et al (1989) utilisant le milieu Palcam.

Les essais montrent une excellente concordance entre les méthodes mais une sensibilité et surtout une rapidité de résultats supérieure avec le milieu Palcam.
\end{abstract}

Listeria / Palcam / rapidité de réponse / dénombrement

Summary - Isolation and counting of Listeria in milk products. The systemization of Listeria research in pathological, nutritious and especially milk products led to the optimization of methods of detection and counting of these bacteria. In this study, we compared the respective performances of the method recommended by the FDA taking as basis the work of Lovett (1988), and that of Van Netten et al (1989), using the Palcam medium. The tests show excellent agreement between the 2 methods, but a higher sensitivity and more rapid results with the Palcam medium.

Listeria / Palcam / rapidity of response / counting

\section{INTRODUCTION}

L'observation de flambées épidémiques de listériose, en particulier sur le continent américain, a permis de franchir une étape décisive en mettant en évidence le rôle de l'aliment dans la contamination humaine, battant ainsi en brèche la notion de zoonose qui prévalait encore il y a peu de temps. Ce regain d'intérêt pour la recherche et la lutte contre Listeria a profondément modifié les besoins en techniques analytiques. On est, en effet, passé du domaine de la pathologie à celui de l'environ- nement et de l'hygiène alimentaire ce qui impose à l'homme de laboratoire des difficultés supplémentaires. II traite, en effet, non plus des prélèvements pathologiques, en général monomicrobiens et souvent très contaminés mais des produits, dans un grand nombre de cas, peu riches en Listeria au stade où l'analyse est effectuée, quelquefois polymicrobiens, susceptibles d'évoluer, surtout s'ils sont réfrigérés, et pour lesquels un résultat précis et rapide doit être formulé, surtout si l'on se place dans le cadre d'une exportation ou d'une commercialisation. À ces difficultés s'ajoutent celles du dénombrement des 
Listeria pourtant riche en enseignements tant dans le domaine de l'hygiène alimentaire que dans celui de l'étude des modes de contamination et de transmission de la maladie.

Le protocole mis au point par cette étude a l'avantage de s'appliquer à une très large variété d'échantillons, qu'il s'agisse de produits alimentaires, carnés ou laitiers, ou encore de produits pathologiques. Les échantillons ne subissent de préparation particulière que principalement dans deux cas :

- lorsque les aliments ont subi des traitements thermiques ou s'ils ont été soumis à des $\mathrm{pH}$ extrêmes, il est conseillé de mettre en œuvre les techniques de revivification décrites par Mossel et al (1984) ce qui, dans ce cas, exclut la possibilité d'effectuer des dénombrements,

- il s'avère quelquefois indispensable d'ajuster à 7 , le $\mathrm{pH}$ de certains fromages, par adjonction de quelques gouttes de soude normale, de manière à écarter les possibilités d'obtenir un résultat faussement négatif, et ce, quelle que soit la technique utilisée.

\section{MATÉRIEL ET MÉTHODE}

L'étude porte sur 664 échantillons de lait de mélange en provenance de tanks de producteurs et sur 991 fromages d'origines variées : pâtes molles au lait cru ou pasteurisé, pâtes molles à croûtes lissées ou frottées, fromages fondus au lait pasteurisé, pâtes pressées demi-cuites ou non cuites, fromages de chèvre à pâte molle au lait cru ou pasteurisé.

\section{Matériel}

L'utilisation d'un broyeur de type «Stomacher» est nécessaire pour l'homogénéisation optimale de certains échantillons (fromages, par exemple).

\section{Méthodes}

\section{Composition des milieux de culture}

Technique «FDA modifiée», selon la note
du ministère de l'Agriculture, 1987

— bouillon d'enrichissement sélectif (EB)

Préparer le bouillon trypticase soja en suivant les instructions du fabricant et ajouter $6 \mathrm{~g} / \mathrm{d}$ dextrait de levure; le pH doit être de 7,3 à $25^{\circ} \mathrm{C}$ après stérilisation.

Répartir le bouillon dans des récipients de $500 \mathrm{ml}$ de telle sorte que le volume soit de 225 $\mathrm{ml}$ après stérilisation.

Au moment de l'emploi, ajouter :

- solution à $0,75 \%$ d'acriflavine $0,5 \mathrm{ml}$;

— solution à $2 \%$ d'acide nalidixique $0,5 \mathrm{ml}$;

— solution à $2,5 \%$ de cycloheximide $0,5 \mathrm{ml}$

(conservation possible 1 semaine aux environs de $5^{\circ} \mathrm{C}$ );

bien mélanger après chaque addition.

- gélose sélective Mac Bride modifiée (MMA)

- phényl éthanol agar $35,5 \mathrm{~g}$; glycine anhydre $10 \mathrm{~g}$; chlorure de lithium $0,5 \mathrm{~g}$; eau distillée $1 \mathrm{I}$.

Le $\mathrm{pH}$ doit être de 7,3 à $25^{\circ} \mathrm{C}$ après stérilisation.

Répartir le milieu gélosé de telle sorte que le volume soit de $100 \mathrm{ml}$ après stérilisation.

Au moment de l'emploi, après fusion du milieu et refroidissement à $45^{\circ} \mathrm{C}$ au bain d'eau, ajouter :

- solution à 2,5\% de cycloheximide $0,8 \mathrm{ml}$.

Bien mélanger et répartir à raison de $15 \mathrm{ml}$ dans des boîtes de Petri de $90-100 \mathrm{~mm}$ de diamètre. Ne pas sécher. Conservation 1 semaine au réfrigérateur.

\section{Milieux de culture de la méthode Palcam}

- bouillon d'enrichissement sélectif (EB) Cf paragraphe précédent;

- milieu Palcam (selon Van Netten et al, 1989)

peptone $23,0 \mathrm{~g}$; amidon 1,0 g; chlorure de sodium $5,0 \mathrm{~g}$; mannitol $10,0 \mathrm{~g}$; citrate de fer ammoniacal $0,5 \mathrm{~g}$; esculine $0,8 \mathrm{~g}$; glucose $0,5 \mathrm{~g}$; chlorure de lithium $15,0 \mathrm{~g}$; rouge de phénol $0,08 \mathrm{~g}$; agar agar $13 \mathrm{~g}$; eau déminéralisée $1000 \mathrm{ml}$. 
Le pH est ajusté à $7,2 \pm 0,1$, le milieu est autoclavé $15 \mathrm{~min}$ à $121^{\circ} \mathrm{C}$ puis ramené à $50^{\circ} \mathrm{C}$, température à laquelle sont ajoutés : sulfate de polymyxine B $10,0 \mathrm{mg}$; ceftazidime $20,0 \mathrm{mg}$ et acriflavine $5,0 \mathrm{mg}$.

Le milieu est ensuite coulé en boîtes de Petri de $90 \mathrm{~mm}$ de diamètre.

Après incubation à $37^{\circ} \mathrm{C}$ pendant $24-48 \mathrm{~h}$, les colonies de Listeria sont facilement repérables à l'œil nu. De couleur marron vert foncé, elles présentent une petite dépression centrale et sont légèrement incrustées dans la gélose. Lorsque l'inoculum est suffisamment contaminé, elles sont visibles 4 fois sur 5 au bout de $24 \mathrm{~h}$ d'incubation. Le dénombrement est possible à partir de boîtes directement ensemencées. Un des intérêts de ce milieu réside dans la présence d'esculine et de mannitol. Les colonies de Listeria (esculine + mannitol -) apparaissent entourées d'un halo noir dû à l'attaque de l'esculine, alors que la présence éventuelle d'entérocoques (esculine + mannitol +) se repère par un noircissement des colonies et un virage du rouge de phénol, provoquant une coloration jaune diffuse, révélant ainsi l'attaque du mannitol.

\section{Protocole «FDA modifié»}

Au temps $t, 25 \mathrm{~g}$ (ou $25 \mathrm{ml}$ ) d'échantillon sont homogénéisés dans $225 \mathrm{ml}$ de bouillon EB puis incubés à $30^{\circ} \mathrm{C}$. Au temps $t+24 \mathrm{~h}, t+48 \mathrm{~h}, t+$ $7 \mathrm{j}$, on isole en stries sur gélose MMA une anse bouclée de bouillon enrichi ainsi qu'une anse bouclée d'une dilution au $1 / 10$ en $\mathrm{KOH}$ à $0,5 \%$ du même bouillon.

Parallèlement, au temps $t+24 \mathrm{~h}$, on ensemence $0,1 \mathrm{ml}$ de bouillon dans $10 \mathrm{ml}$ d'un bouillon EB que l'on incube à $30^{\circ} \mathrm{C}$ pendant $24 \mathrm{~h}$ avant repiquage sur gélose MMA.

Après incubation des géloses MMA à $35^{\circ} \mathrm{C}$ pendant $48 \mathrm{~h}$, on repère les colonies par la technique de transillumination oblique de Henry (1933).

Les colonies suspectes sont repiquées et identifiées.

\section{Méthode Palcam}

\section{Laits (fig 1)}

- Au temps $t, 0,2 \mathrm{ml}$ de lait sont directement étalés au rateau sur une gélose Palcam et incubés à $37^{\circ} \mathrm{C}$. Deux lectures sont effectuées respectivement à 24 et $48 \mathrm{~h}$;

- parallèlement, un volume minimal de $30 \mathrm{ml}$ de lait est dilué au 1/2 en bouillon d'enrichissement et incubé à $37^{\circ} \mathrm{C}$ pendant $7 \mathrm{j}$;

- un isolement de ce bouillon est effectué sur gélose Palcam aux temps $t+24 \mathrm{~h}$ et $t+7 \mathrm{j}$, incubé à $37^{\circ} \mathrm{C}$ et lu après 24 et $48 \mathrm{~h}$.

\section{Fromages (fig 2)}

- Au temps $t, 100 \mathrm{~g}$ de fromage sont homogénéisés à l'aide d'un broyeur de type "Stomacher» dans $200 \mathrm{ml}$ de bouillon d'enrichissement à partir desquels $0,2 \mathrm{ml}$ sont prélevés et immédiatement étalés au rateau sur une gélose Palcam incubée à $37^{\circ} \mathrm{C}$ et lue au bout de 24 et $48 \mathrm{~h}$.

Dans l'optique d'un dénombrement, on ensemence en plus, et selon le même protocole $0,2 \mathrm{ml}$ de 3 dilutions successives $10^{-1}, 10^{-2}$ et $10^{-3}$ de ce $\mathrm{L}$ - uillon d'enrichissement à raison de $1 \mathrm{ml}$ dans $9 \mathrm{ml}$ d'une solution stérile de $\mathrm{KOH}$ à $0,5 \%$ dans l'eau déminéralisée.

Le bouillon d'enrichissement est ensuite incubé à $37^{\circ} \mathrm{C}$ pendant $7 \mathrm{j}$.

Comme pour le lait, un isolement de ce bouillon est effectué sur gélose Palcam à $t+$ $24 \mathrm{~h}$ et $t+7 \mathrm{j}$, incubé et lu selon le même protocole.

\section{Identification des souches}

Quelle que soit la technique utilisée (FDA modifiée ou Palcam), l'isolement des colonies et l'identification des souches sont indispensables. Dans les 2 cas, on se réfère aux modalités décrites dans la technique FDA modifiée. 


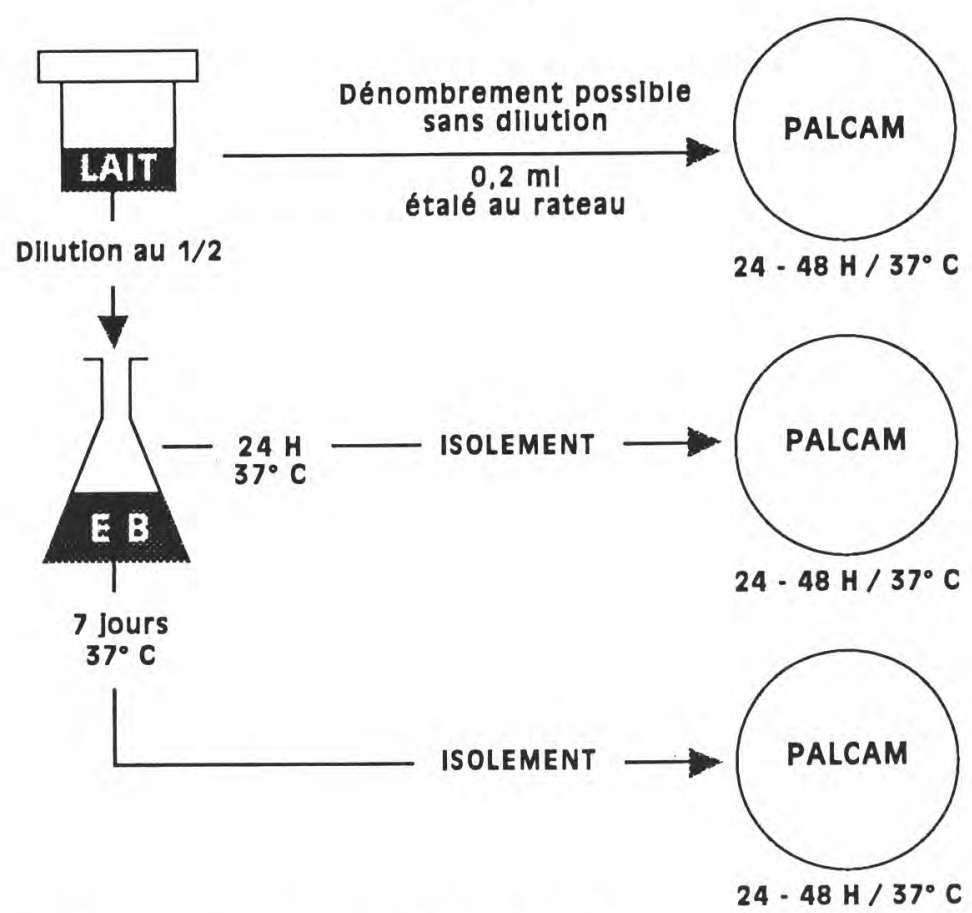

Fig 1. Isolement et dénombrement de Listeria à partir du lait.

Isolation and enumeration of Listeria from milk.

\section{RÉSULTATS - COMPARAISON AVEC LA TECHNIQUE FDA}

Cette comparaison a été effectuée à partir de 1655 échantillons comprenant 664 laits de mélange et 991 fromages dont certains proviennent de laiteries confrontées à des problèmes de contamination par Listeria.

\section{Résultats globaux}

Ces résultats sont publiés dans le cadre d'une étude comparative, réalisée à partir d'un échantillonnage non représentatif de l'ensemble des produits laitiers vis-à-vis desquels toute extrapolation serait abusive.

Les figures 3 et 4 expriment les résultats globaux, toutes techniques confondues.

\section{Etude de la concordance des 2 techniques}

Le tableau I, établi à partir du cumul des résultats positifs et la figure 5 indiquent tous 2 une excellente concordance entre les méthodes :

- on observe que, parmi les résultats positifs, environ $9 \%$ le sont uniquement grâce au milieu Palcam; 


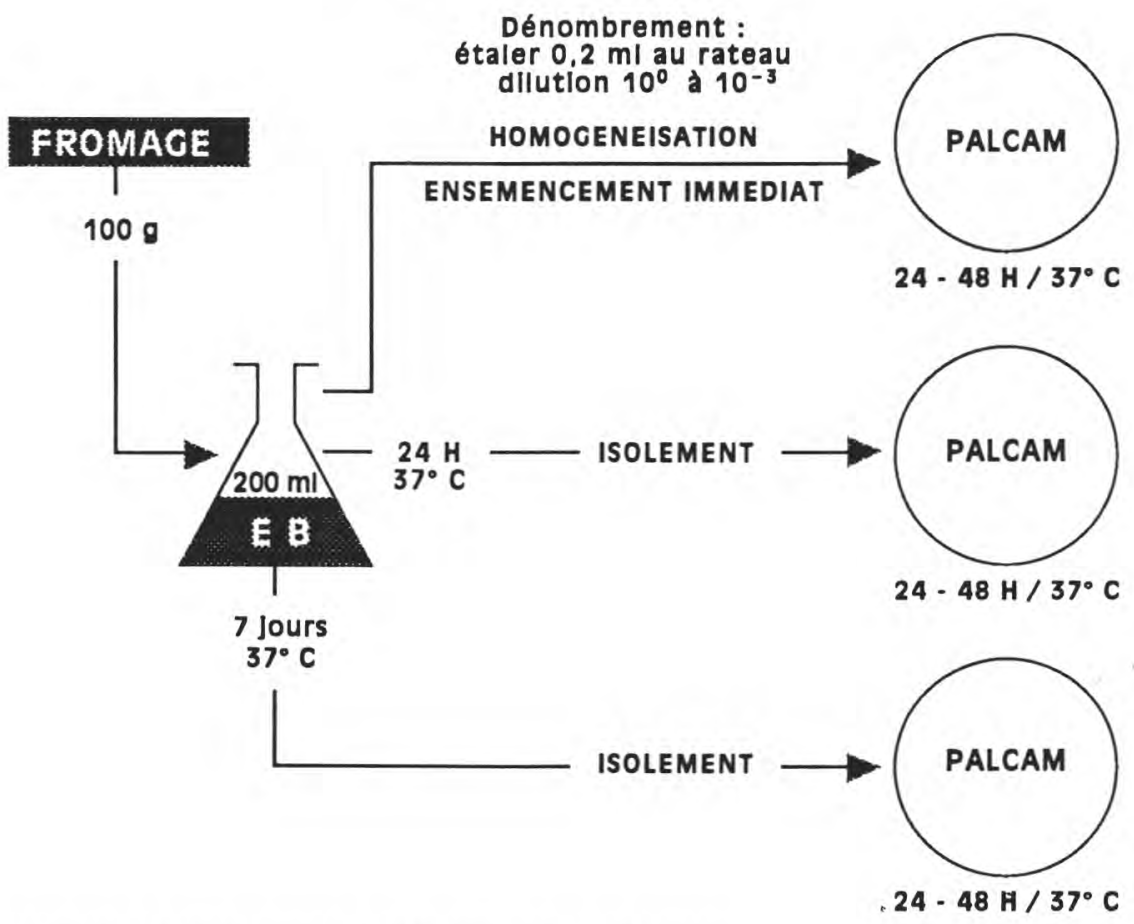

Fig 2. Isolement et dénombrement de Listeria à partir de fromage Isolation and enumeration of Listeria from cheese.

- quant aux Listeria $(0,68 \%)$ retrouvées uniquement par la technique FDA, il faut noter qu'elles présentaient certaines particularités rendant leur identification douteuse.

\section{Comparaison de la rapidité des techniques}

Le tableau II montre à l'évidence que l'utilisation du milieu Palcam diminue très notablement le temps de réponse.

Plus de $60 \%$ des résultats positifs sont obtenus en 24 h et plus de $90 \%$ le sont en $48 \mathrm{~h}$, ce qui surpasse en rapidité d'exécution la plupart des protocoles actuellement utilisés.

\section{Dénombrement}

À partir des laits de mélange, les résultats font état en général d'un taux de contamination inférieur à 100 colonies revivifiables par $\mathrm{ml}$. En ce qui concerne la majorité des fromages étudiés, le taux de contamination se situe entre 20 et $10^{4}$ colonies par g. II est possible de dénombrer dans quelques fromages jusqu'à $10^{5}$ colonies par $\mathrm{g}$.

Exceptionnellement, un fromage contenait $3 \times 10^{6}$ colonies de Listeria par $\mathrm{g}$.

\section{Observation des contaminations multiples sur milieu Palcam}

A la lumière de cette étude, il semble plus facile de déceler une contamination mul- 


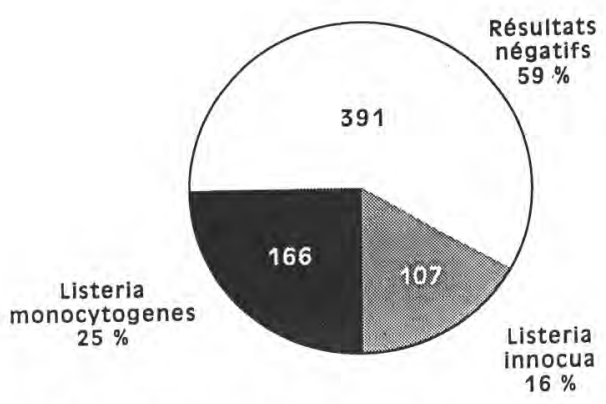

Fig 3. Résultats obtenus à partir de 664 échantillons de lait.

Results from 664 milk samples.

tiple par différents types de Listeria à partir des ensemencements directs par rapport à ce qui est observé après enrichissement.

À titre d'exemple, le typage de 5 souches isolées directement à partir d'un fromage, a montré la présence de 2 sérotypes de Listeria monocytogenes se répartissant en 4 lysotypes différents. Après enrichissement, il n'était plus possible de retrouver cette même variété.

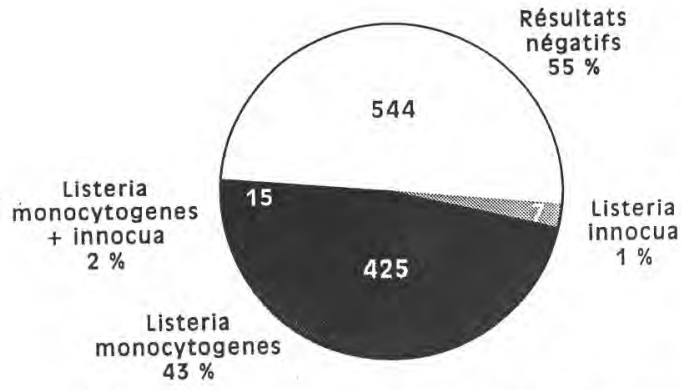

Fig 4. Résultats obtenus à partir de 991 échantillons de fromages.

Results from 991 cheese samples.

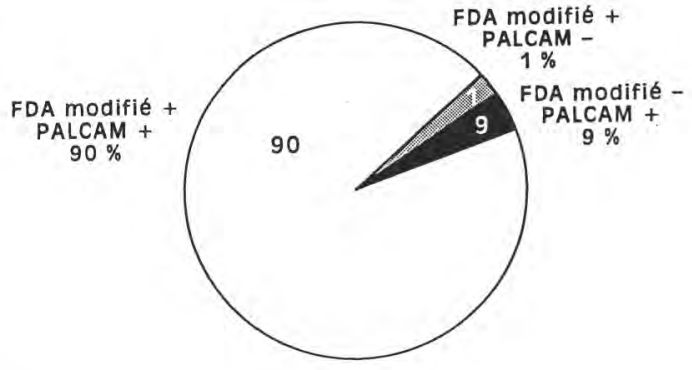

Fig 5. Concordance des techniques. Agreement between the methods.

\section{CONCLUSION}

Le protocole Palcam présente de nombreux avantages :

- simplicité d'exécution, rapidité dans la réponse;

- sensibilité accrue par rapport à la technique «FDA modifiée";

- domaines d'utilisation variés :

- analyse des points critiques et contrôle de la qualité des denrées en industries agro-alimentaires;

- recherche fondamentale : études épidémiologiques, écopathologie, étude de la transmission et de la pathogénie de la maladie, détermination de la dose minimale infectante concernant les aliments destinés à la consommation humaine;

- contrôle officiel des denrées alimentaires pour lesquelles un dénombrement peut s'avérer indispensable lorsqu'il sera possible de fixer des taux maximaux admissibles de contamination des produits alimentaires par Listeria. 
Tableau I. Taux de concordance des 2 techniques, établi à partir du cumul des résultats positifs. Agreement rate between the 2 methods from the total of positive results.

\begin{tabular}{lccc}
\hline Technique & Résultats & $\begin{array}{c}\text { Nombre } \\
\text { d'échantillons }\end{array}$ & Concordance \\
\hline $\begin{array}{l}\text { FDA modifié } \\
\text { Palcam }\end{array}$ & + & 664 & $90,34 \%$ \\
FDA modifié & + & 66 & $8,98 \%$ \\
$\begin{array}{l}\text { Palcam } \\
\text { FDA modifié }\end{array}$ & + & 5 & $0,68 \%$ \\
Palcam & + & & \\
\hline
\end{tabular}

Tableau II. Comparaison de rapidité des techniques Palcam et FDA modifiée à partir du cumul des résultats trouvés positifs par les 2 techniques.

Comparison of the rapidity of the Palcam and modified FDA methods based on total of positive results found by the 2 techniques.

\begin{tabular}{|c|c|c|c|c|c|c|}
\hline \multirow[b]{2}{*}{ Lecture } & \multicolumn{3}{|c|}{ Palcam } & \multicolumn{3}{|c|}{ FDA modifié } \\
\hline & $\begin{array}{l}\text { Résultat } \\
\text { positif }\end{array}$ & $\%$ & $\begin{array}{l}\text { Cumul } \\
t+48 h\end{array}$ & $\begin{array}{l}\text { Résultat } \\
\text { positif }\end{array}$ & $\%$ & $\begin{array}{l}\text { Cumul } \\
t+48 h\end{array}$ \\
\hline$t+24 \mathrm{~h}$ & 419 & 63,10 & & 0 & 0,00 & \\
\hline$t+48 \mathrm{~h}$ & 187 & 28,16 & $91,26 \%$ & 0 & 0,00 & $0,00 \%$ \\
\hline$t+72 \mathrm{~h}$ & 0 & 0,00 & & 494 & 74,40 & \\
\hline$t+8 \mathrm{j}$ & 58 & 8,74 & & 170 & 25,60 & \\
\hline
\end{tabular}

\section{REMERCIEMENTS}

À S Artault, B. Volant et C Ternant pour leur collaboration technique.

\section{RÉFÉRENCES}

Henry BS (1933) Dissociation in the genus Brucella. J Infect Dis 52, 374-402

Lovett J (1988) Isolation and identification of Listeria monocytogenes in dairy products. J Assoc Off Anal Chem 71, 658-660
Ministère Agriculture (1987) Etablissements de fabrication de fromages : recherche de Listeria monocytogenes; agrément pour l'exportation. Note Service DG-AL/SVHA/N8/N 8041

Mossel DAA, Van Netten P (1984) Harmful effects of selective media on stressed microorganisms: nature and remedies. In: The revival of injured microbes. (Andrew MHE, Russel AD, eds). Acad Press, London, 329-369

Van Netten P, Perales I, Van de Mossdijk A, Curtis GDW, Mossel DAA (1989) Liquid and solid selective differential media for the detection and enumeration of $L$ monocytogenes and other Listeria spp. Int J Food Microbiol 8, 299-316 